\title{
Long-acting injectable paliperidone palmitate for schizophrenia and alcohol use disorder
}

\begin{abstract}
Alcohol use disorder (AUD) worsens the course of schizophrenia. Its treatment is with antipsychotics, mainly clozapine. However, long-acting injectable (LAI) antipsychosis second generation (ASG) also shows improvement in the reduction of alcohol consumption in patients with schizophrenia. We have two clinical cases, a woman and a man, both with schizophrenia and AUD that after treatment with paliperidone palmitate depot monthly. The psychopathology improved and reduction of alcohol consumption was observed in one of the cases, by monotherapy with paliperidone palmitate monthly and, complete abstinence in the other case, by polytherapy with clozapine and paliperidone palmitate monthly.
\end{abstract}

Keywords: antipsychosis, paliperidone palmitate, long-acting injectable, schizophrenia, alcohol
Volume 6 Issue 2 - 2018

\section{Lopez Arteaga T \\ Service of Psychiatric, Institute of Health Sciences of Castilla La-Mancha, Spain}

Correspondence: Lopez Arteaga T, Service of Psychiatric, Institute of Health Sciences of Castilla La-Mancha, Carretera de Madrid S/N, 45600, Talavera de la Reina, Spain,

Email mteresala@sescam.jccm.es

Received: February 24, 2018| Published: March 16, 2018
Abbreviations: ABU, addictive behaviors unit; $\mathrm{AFG}$, antipsychotic first generation; ASG, antipsychotic second generation; AUD, alcohol use disorder; ER, emergency room; LAI, long-acting injectable; SUD, substance use disorders

\section{Introduction}

We present two clinical cases in which, after the use of paliperidone palmitate depot monthly has been reduced, and even abandoned the consumption of alcohol in two patients, man and woman, both with schizophrenia and alcohol use disorder (AUD). The information was obtained through clinical interview, clinical history and weekly urine toxic controls in the last three years.

\section{Case |}

A 48-year-old woman without medical history of interest or any psychiatric family background, diagnosed with Schizophrenia and AUD since 1992. Until 2016, she had several urgent and scheduled hospital admissions, a parallel follow-up with the addictive behaviors unit (ABU) and finally, she had to be legally incapacitated, setting his mother as legal guardian and the patient needed to check-in on a community residential center. Throughout its evolution it has had different antipsychotic treatments, maintaining in the last 4 years treatment with clozapine. In 2015, he had 7 (Emergency Room) ER check-ins for alcohol intoxication. These intoxications occurred both in the context of psychopathological exacerbation with disorganization of thought, and in periods of stability of the psychotic clinic. After her last admission in 2016, she has been treated with clozapine $400 \mathrm{mg} /$ daily, aripiprazole $10 \mathrm{mg}$ /daily and flufenazin decanoate $25 \mathrm{mg}$ every 14 days. Two months later, the patient and her legal guardian were proposed to change depot antipsychotic treatment. They both preferred to maintain polytherapy to increase clozapine dose (consent signed by the tutor) prescribing clozapine $400 \mathrm{mg} / \mathrm{d}$ and paliperdione palmitate at a rate of $150 \mathrm{mg} / \mathrm{monthly}$. Flufenazin decanoate was discontinued, as well as aripiprazole. After one month with this treatment the patient presaged complete abstinence to alcohol, (which we confirm through negative urine toxic controls), which she has maintained since then. She did not need to go to the emergency room or to enter the hospital due to psychopathological de-compensation during this period. In addition, in the last 6 months, before the stability and clinical improvement, the total neuroleptic dose was reduced, taking the patient at the present time clozapine $400 \mathrm{mg} / \mathrm{d}$ and palmitate paliperidone monthly $75 \mathrm{mg}$. (Clozapine levels in blood according to the latest measurement $389 \mathrm{ng} / \mathrm{ml}$ with total oral dose of $400 \mathrm{mg} / \mathrm{d}$ ).

\section{Case 2}

A 59-year-old male patient, diagnosed with paranoid schizophrenia and AUD. In 2013, he had hospital admission and at discharge he was treated with flufenazin decanoate $25 \mathrm{mg} / \mathrm{ml}$ every 3 weeks and risperidone $6 \mathrm{mg} /$ day orally. Gradually, risperidone was removed due to stability in the psychotic clinic, but alcohol consumption was maintained, with frequent intoxications. These episodes of abuse were maintained both with flufenazin decanoate and risperidone combination therapy. In 2016, it was replaced (Antipsychotic Second Generation) AFG with ASG depot, and Paliperidone palmitate $150 \mathrm{mg} / \mathrm{monthly}$ was introduced. After 2 months of treatment, the patient reported a sustained consumption of alcohol in a much smaller amount than in previous years (between 1-2UBE day), without reaching intoxication.

\section{Discussion}

The lifetime frequency of substance use disorders (SUD) in patients with schizophrenia is almost $50 \%$. Nicotine, alcohol and cannabis are the substances that are used most frequently. ${ }^{1}$ AUD usually occurs in patients with schizophrenia, contributes greatly to their morbidity ${ }^{2}$ and drastically increased their course. ${ }^{3}$ There are few data available on pharmacological approaches in this population, but among antipsychotics, clozapine shows positive evidence in the literature, ${ }^{4}$ it has been associated with a decrease in alcohol consumption in patients with schizophrenia, but its toxicity reduces its use. ${ }^{3}$ In addition, this group of patients often show poor adherence to medication. To improve substance consumption and adherence to treatment in schizophrenic patients with SUD, direct and indirect evidence tends to favor the use of second generation antipsychotics (SGA), particularly those with less metabolic effects, cardiovascular and extra pyramidal, as well as those with their depot formulation. ${ }^{5,6}$ Studies have been conducted with oral SGA and LAI to assess the 
decrease in alcohol consumption in patients with schizophrenia. Some of these studies have been carried out with risperidone, aripiprazole, paliperidone and iloperidone. . $^{1,37-10}$

Chau et al. ${ }^{8}$ propose the unique and broad pharmacology of clozapine profile, including its weak dopamine $\mathrm{D}_{2}$ receptor blockade, its potent antagonism of the noradrenergic receptor alpha-2, and its ability to elevate chronically and highly. Norepinephrine may have the property of reducing alcohol abuse. Risperidone is an atypical antipsychotic with pharmacological properties partially overlapping those of clozapine. Like clozapine, risperidone is an antagonist of dopamine $\mathrm{D}_{2}$ receptors and noradrenergic receptors alpha-2. Its metabolite paliperidone (9-OH-risperidone) has a similar affinity for alpha-2 receptors but low affinity (and rapidity of binding) for the $\mathrm{D}_{2}$ receptors that risperidone, which makes it more similar to clozapine in its actions on these receptors. Risperidone, however, may differ from clozapine in regard to norepinephrine reuptake, since it does not appear to raise norepinephrine levels in patients with schizophrenia to the same extent as clozapine, however paliperidone may have some effects similar to those of norepinephrine reuptake in vivo. ${ }^{3}$

According to Green et al. ${ }^{10}$ patients with schizophrenia with alcohol use disorder seem to continue the consumption of alcohol while taking any form of risperidone. However, their data suggest that risperidone injection may be a better option than oral form for these patients with double diagnosis. ${ }^{10}$ According to Chau et al. ${ }^{8}$ paliperidone itself does not chronically reduce the consumption of alcohol, but is able to do so when it is administered in conjunction with the norepinephrine reuptake inhibitor desipramine. On the basis of current data, they speculate that patients with schizophrenia with comorbid alcohol consumption disorder may benefit from the use of paliperidone in combination with desipramine. Their data suggest that this combination of drugs, which exhibits inhibition of norepinephrine reuptake, potent blockade of norepinephrine alfa- 2 receptors and relatively modest dopamine $\mathrm{D}_{2}$ blockade receptors may be useful (and should be tested) in the treatment of the disorder by alcohol consumption in patients with schizophrenia, and potentially those with alcohol use disorder alone. ${ }^{3}$

The iloperidone studied in the animal model decreases the consumption of alcohol with the same efficacy as clozapine, and desipramine seems to amplify this effect. The data suggest that iloperidone, alone or in combination with desipramine, should be analyzed in patients with schizophrenia and alcohol use disorder. ${ }^{1}$ Aripiprazole was the first antipsychotic developed to possess agonist properties at dopamine $\mathrm{D}_{2}$ autoreceptors, a groundbreaking strategy that presented a new vista for schizophrenia drug discovery. The dopamine $\mathrm{D}_{2}$ receptor is the crucial target of all extant antipsychotics, and all developed prior to aripiprazole were $\mathrm{D}_{2}$ receptor antagonists. Extensive blockade of these receptors, however, typically produces extra pyramidal (movement) side effects which plagued firstgeneration antipsychotics, such as haloperidol. Second-generation antipsychotics, such as clozapine, with unique Polypharmacology and $\mathrm{D}_{2}$ receptor binding kinetics, have significantly lower risk of movement side effects, but can cause myriad additional ones, such as severe weight gain and metabolic dysfunction. Aripiprazole (characterized by its unique agonist activity at dopamine $\mathrm{D}_{2}, \mathrm{D}_{3}$ and serotonin $5-\mathrm{HT}_{1 \mathrm{~A}}$ receptors as well as antagonist activity at serotonin $5-\mathrm{HT}_{2 \mathrm{~A}}$ receptors) translates to successful reduction of positive, negative, and cognitive symptoms of schizophrenia, while also mitigating risk of weight gain and movement side effects. ${ }^{11}$ However, the use of aripiprazol in AUD cannot be drawn in patients with schizophrenia. ${ }^{9}$

\section{Conclusion}

With the clinical cases shown and the referred evidence, we can draw the following conclusions:

a. LAI paliperidone palmitate can by itself reduce the consumption of alcohol in schizophrenia, as it is the case in case 2 .

b. Clozapine did not reduce the consumption of alcohol in case 1, but in association with paliperidone. This may be due to paliperidone's own efficacy in reducing alcohol consumption, to the fact that clozapine levels were below the therapeutic level, or because the reduction in consumption was due to polytherapy.

c. In any case, they do reflect both cases that the use of long-acting ASG reduces the consumption of alcohol and schizophrenia, in addition, although they improve the adherence, the clinical stabilization and the associated substances used.

d. In the case of monotherapy with paliperidone palmitate long acting, the consumption of alcohol has been reduced (case 2) and in the case of polytherapy, completes remission has been achieved (case 1). We cannot affirm that these facts are due to gender differences, antipsychotic association or by the patient's own decision to maintain active consumption or not.

In the future, studies should be carried out to evaluate the effectiveness of clozapine versus long-acting injectable ASG in schizophrenia and alcohol consumption.

\section{Acknowledgement}

None.

\section{Conflict of interest}

None.

\section{References}

1. Khokhar JY, Green AI. Effects of iloperidone, combined with desipramine, on alcohol drinking in the Syrian golden hamster. Neuropharmacology. 2016;105:25-34

2. Khokhar JY, Todd TP. Behavioral predictors of alcohol drinking in a neurodevelopmental rat model of schizophrenia and co-occurring alcohol use disorder. Schizophr Res. 2017;194:91-97.

3. Chau DT, Green AI, Khokhar JY, et al. Desipramine enhances the ability of paliperidone to decrease alcohol drinking. J Psychiatr Res.2015;69:9-18.

4. Arranz B, Garriga M, Garcia-Rizo C, et al. Clozapine use in patients with schizophrenia and a comorbid substance use disorder: A systematic review. Eur Neuropsychopharmacol. 2018;28(2):227-242.

5. Azorin JM, Simon N, Adida M, et al. Pharmacological treatment of schizophrenia with comorbid substance use disorder. Expert Opin Pharmacother. 2016;17(2):231-253.

6. Werner FM, Covenas R. Long-term administration of antipsychotic drugs in schizophrenia and influence of substance and drug abuse on the disease outcome. Curr Drug Abuse Rev. 2017;10. 
7. Lynn Starr H, Bermak J, Mao L, et al. Comparison of long-acting and ora antipsychotic treatment effects in patients with schizophrenia, comorbid substance abuse, and a history of recent incarceration: An exploratory analysis of the PRIDE study. Schizophr Res. 2017;194:39-46.

8. Chau DT, Khokhar JY, Gulick D, et al. Desipramine enhances the ability of paliperidone to decrease alcohol drinking. J Psychiatr Res. 2015;69:9-18.
9. Brunetti M, Di Tizio L, Dezi S, et al. Aripiprazole, alcohol and substance abuse: a review. Eur Rev Med Pharmacol Sci. 2012;16(10):1346-1354.

10. Green AI, Brunette MF, Dawson R, et al. Long-acting injectable vs oral risperidone for schizophrenia and co-occurring alcohol use disorder: a randomized trial. J Clin Psychiatry. 2015;76(10):1359-1365.

11. Casey AB, Canal CE. Classics in Chemical Neuroscience: Aripiprazole. ACS Chem Neurosci. 2017;8(6):1135-1146. 